\title{
Erratum to: Oxygen and sulfur heterocyclic compounds
}

\section{Perspectives on the energetics and structural relationships}

\author{
Vera L. S. Freitas ${ }^{1}$ - Maria D. M. C. Ribeiro da Silva ${ }^{1}$
}

Published online: 6 October 2015

(C) Akadémiai Kiadó, Budapest, Hungary 2015

\section{Erratum to: J Therm Anal Calorim (2015) 121:1059-1071 DOI 10.1007/s10973-015-4800-0}

In the first row of the second column of Tables 3-5, the formula $\Delta_{\mathrm{f}} H_{\mathrm{m}}^{\mathrm{o}}(\mathrm{g}$, exp) should be corrected to $\Delta_{\mathrm{f}} H_{\mathrm{m}}^{\mathrm{o}}(\mathrm{g}$, exp/comp $)$. The corrected lines are listed below.

Table 3 Comparison of experimental/computational and estimated gas-phase enthalpies of formation of five-membered ring compounds containing oxygen or sulfur heteroatoms

\begin{tabular}{lccc}
\hline $\begin{array}{l}\text { Heteroatom- } \\
\text { containing } \\
\text { compounds }\end{array}$ & $\begin{array}{c}\Delta_{\mathrm{f}} H_{\mathrm{m}}^{\mathrm{o}}(\mathrm{g}, \text { exp/comp }) / \\
\mathrm{kJ} \mathrm{mol}^{-1}\end{array}$ & $\begin{array}{c}\Delta_{\mathrm{f}} H_{\mathrm{m}}^{\mathrm{o}}(\mathrm{g}, \text { estimated })^{a} / \\
\mathrm{kJ} \mathrm{mol}^{-1}\end{array}$ & $\Delta^{\mathrm{b}} /$ \\
$\mathrm{kJ} \mathrm{mol}^{-1}$ \\
\end{tabular}

Table 4 Comparison of experimental/computational and estimated gas-phase enthalpies of formation of six-membered ring compounds containing oxygen or sulfur heteroatoms

\begin{tabular}{cccc}
\hline $\begin{array}{l}\text { Heteroatom- } \\
\text { containing } \\
\text { compounds }\end{array}$ & $\begin{array}{c}\Delta_{\mathrm{f}} H_{\mathrm{m}}^{\mathrm{o}}(\mathrm{g}, \text { exp/comp }) / \\
\mathrm{kJ} \mathrm{mol}^{-1}\end{array}$ & $\begin{array}{c}\Delta_{\mathrm{f}} H_{\mathrm{m}}^{\mathrm{o}}(\mathrm{g}, \text { estimated })^{a} / \\
\mathrm{kJ} \mathrm{mol}^{-1}\end{array}$ & $\Delta^{\mathrm{b}} /$ \\
& & $\mathrm{kJ} \mathrm{mol}^{-1}$ \\
\hline
\end{tabular}

The online version of the original article can be found under doi:10.1007/s10973-015-4800-0.

Maria D. M. C. Ribeiro da Silva

mdsilva@fc.up.pt

1 Centro de Investigação em Química, Department of Chemistry and Biochemistry, Faculty of Science, University of Porto, Rua do Campo Alegre, 687, 4169-007 Porto, Portugal
Table 5 Comparison of experimental/computational and estimated gas-phase enthalpies of formation of compounds containing nitrogen, oxygen or sulfur heteroatoms

\begin{tabular}{lccc}
\hline $\begin{array}{c}\text { Heteroatom- } \\
\text { containing } \\
\text { compounds }\end{array}$ & $\begin{array}{c}\Delta_{\mathrm{f}} H_{\mathrm{m}}^{\mathrm{o}}(\mathrm{g}, \text { exp/comp }) / \\
\mathrm{kJ} \mathrm{mol}^{-1}\end{array}$ & $\begin{array}{c}\Delta_{\mathrm{f}} H_{\mathrm{m}}^{\mathrm{o}}(\mathrm{g}, \text { estimated })^{a} / \\
\mathrm{kJ} \mathrm{mol}^{-1}\end{array}$ & $\Delta^{\mathrm{b}} /$ \\
& & $\mathrm{kJ} \mathrm{mol}^{-1}$ \\
\hline
\end{tabular}

In Table 3, for the compounds $(\mathrm{X}=\mathrm{S})$, 4-methylbenzothiophene (10), 5-methylbenzothiophene (11), 6-methylbenzothiophene (12), 7-methylbenzothiophene (13), 2-methyldibenzothiophene (15) and 3-methyldibenzothiophene (16), the Ref. [56] should be corrected to [55]. The corrected lines are listed in the next page. 


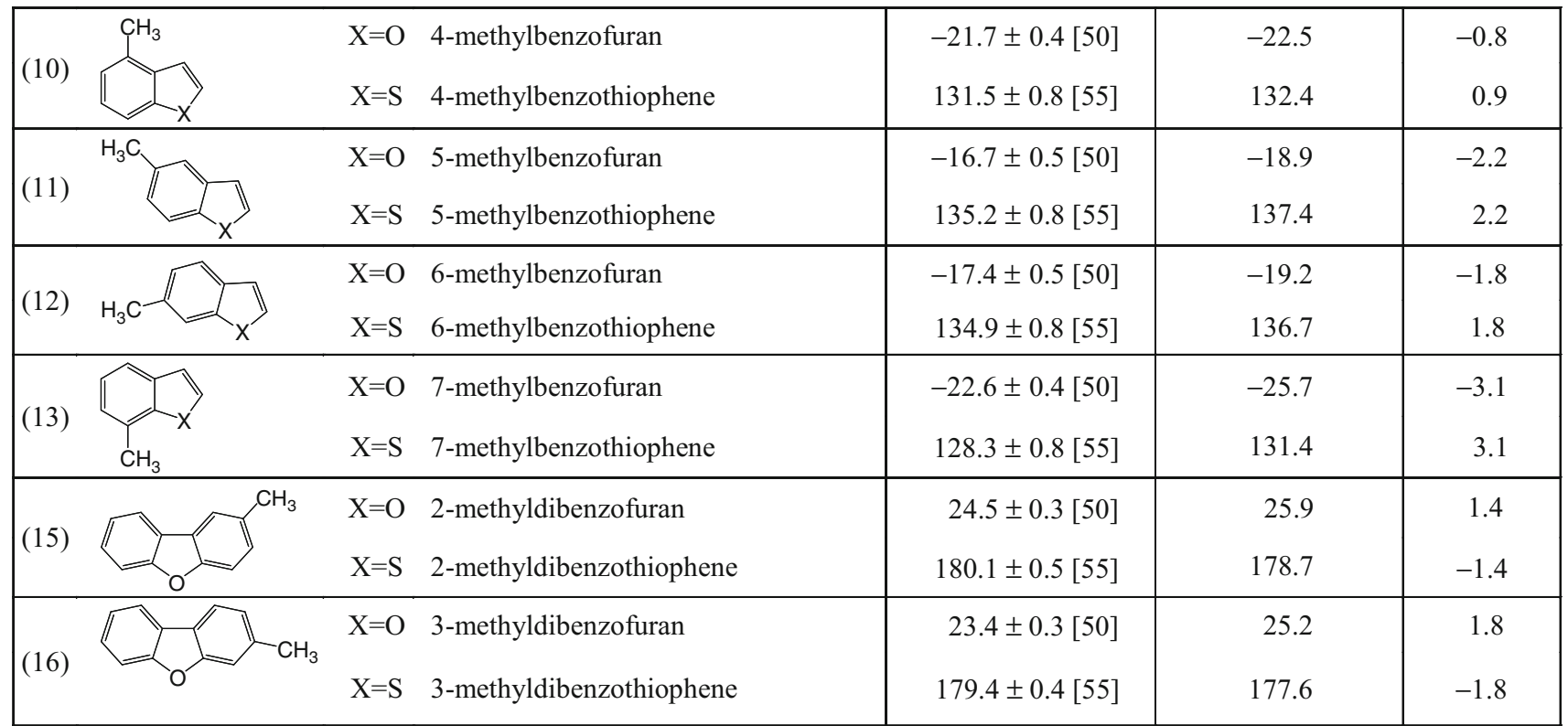

